${ }^{11}$ Gertner, J M, and Lawrie, B, Lancet, 1977, 1, 257.

12 Pietrek, J, et al, Lancet, 1976, 1, 1145.

${ }^{13}$ Cooke, W T, et al, British Medical fournal, 1974, 2, 293.

${ }^{14}$ Hunt, S P, et al, British Medical fournal, 1976, 2, 1351.

15 McCance, R A, and Widdowson, E M, The Composition of Foods, MRC Special Report Series No 297. London, HMSO, 1960.

${ }^{16}$ Department of Health and Social Security, Reports on Public Health and Medical Subjects, No 120. London, HMSO, 1969.

${ }_{17}$ Kirk, T R, Proceedings of the Nutrition Society, 1976, 35, 79A.

${ }^{18}$ Morgan, J, et al, Proceedings of the Nutrition Society, 1976, 35, 74A.
${ }^{19}$ Bansal, R D, et al, Indian fournal of Medical Research, 1973, 61, 1869

20) Karkal, M, Indian Pediatrics, 1975, 12, 13.

21 Department of Health and Social Security, Report on Health and Social Subjects, No 9. London, HMSO, 1974.

2.2 Morgan, $\mathrm{J}$, and Mumford, $\mathrm{P}$, personal communication.

2:3 Jeans, P C, Fournal of the American Medical Association, 1950, 143, 177. ए0

24 Department of Health and Social Security, Report on Health and Social Subjects, No 10. London, HMSO, 1975.

(Accepted 22 December 1977)

\title{
High incidence of vincristine-induced neuropathy in lymphomas
}

\author{
SYLVIA M WATKINS, J P GRIFFIN
}

British Medical fournal, 1978, 1, 610-612

\section{Summary and conclusions}

The incidence of vincristine-induced neuropathy was studied in 60 unselected patients, of whom 23 had lymphoma and 37 had other malignant disease. All were treated with vincristine combined with other cytotoxic agents. Fourteen of the patients with lymphoma $(61 \%)$ developed neuropathy compared with five patients with leukaemia or non-lymphoid cancer $(14 \%)$, even though all patients received comparable doses of vincristine. The difference between the two groups in the incidence of neuropathy was highly significant. Of the patients who developed neuropathy, 17 did so within the first three months of treatment and seven in the first month.

Patients with lymphoma who are receiving vincristine should be observed carefully for symptoms and signs of neuropathy. Vincristine should be withdrawn if progressive neurotoxicity develops.

\section{Introduction}

Peripheral neuropathy is a well-known complication of vincristine treatment. Depression of the ankle-jerk reflex is almost universal, and symptoms of neurotoxicity are extremely common in patients receiving high doses. ${ }^{12}$ Nevertheless, most patients given normal doses develop areflexia and some get paraesthesiae that may be severe enough to warrant stopping treatment. ${ }^{3}$ Motor and autonomic neuropathy may also complicate vincristine treatment. ${ }^{2}$ We decided to compare the incidence of vincristine-induced neuropathy in patients with lymphoma and other malignant diseases.

\section{Patients and methods}

All patients starting treatment with vincristine in our oncology clinic between August 1974 and October 1976 were studied. During this time 60 patients with lymphoma and other malignant disease were started on courses of cytotoxic drugs including vincristine (see

Lister Hospital, Stevenage, Herts SG1 4AB

SYLVIA M WATKINS, DM, MRCP, consultant physician J P GRIFFIN, MB, PHD, clinical pharmacologist table). Twenty-three patients had lymphoma (10 Hodgkin's disease $\bar{\omega}$ and 13 non-Hodgkin's lymphomas), 23 had metastatic breast : cancer, nine had leukaemia (five acute myeloblastic, one acute promyelocytic, one acute myelomonocytic, one blast crisis, and one acute lymphoblastic), and one each had malignant melanoma, rectal carcinoma, $\rightarrow$ bronchial carcinoma, undifferentiated sarcoma, and anaplastic carcinoma. Patients were followed up for a minimum of 10 months or until they died.

Definition of neuropathy-Patients receiving vincristine who com- $\stackrel{\sim}{-}$ plained spontaneously of distressing paraesthesiae with or without $\overrightarrow{0}$ numbness, wasting or muscular weakness, or severe constipation wereoo regarded as having neuropathy. Patients were deliberately not askedర about such symptoms. Areflexia unassociated with symptoms waso disregarded for the purpose of this study.

\section{Results}

INCIDENCE OF NEUROPATHY

Eighteen patients had sensory symptoms and three also had motor symptoms and signs. One patient developed ileus, presumably due to autonomic neuropathy. Ankle jerks were absent in all these 19 patients, and most of them had also lost other reflexes. Vincristine was always stopped when such symptoms developed, and in all cases the symptoms and signs then improved, usually clearing completely 3 . Areflexia alone was not regarded as sufficient reason for stopping vincristine, and indeed almost all the patients in this series had lost at $\frac{0}{3}$ least their ankle jerks.

Fourteen of the 23 patients with lymphoma $\left(61 \%_{0}\right)$ developed $\mathrm{O}$ neuropathy (table). Five of the remaining 37 patients $(14 \%)$ developed N neuropathy, of whom two had leukaemia, one breast cancer, one melanoma, and one anaplastic carcinoma. The difference between the two groups in the incidence of neuropathy was highly significant $\left(\%^{2}=14 \cdot 7 ; \mathrm{DF}=1 ; \mathrm{P}<0.0005\right)$

AGE

The mean age of patients with neuropathy was slightly but not? significantly lower than that of the patients without it $(45 \cdot 1$ and $50 \cdot 3$ years respectively; $t=1 \cdot 228 ; \mathrm{DF}=58 ; \mathrm{P} \cdot 0 \cdot 1)$. Similarly, patients $\overline{0}$ with lymphoma with and without neuropathy were of comparable mean ages $\left(47 \cdot 3\right.$ and $55 \cdot 2$ years respectively; $t=1 \cdot 139 ; \mathrm{DF}=21 ; \frac{\text { }}{\mathbb{D}}$ P. $0 \cdot 1)$, as were the patients with and without lymphoma $(50.4$ and 0 47.6 years respectively; $t=0.683 ; \mathrm{DF}=58 ; \mathrm{P}>0.2)$.

DOSE OF VINCRISTINE

The total dose of vincristine given before neuropathy developed was usually low (mean $0.14 \mathrm{mg} / \mathrm{kg}$ ). In contrast many of the patients without neuropathy had received large cumulative doses (mean $0.36 \mathrm{mg} / \mathrm{kg}$ ), in some cases for prolonged periods. Figs 1 and 2 show 
Clinical details of patients, vincristine doses and duration of treatment, and proportions of patients developing neuropathy during treatment. Values are means (ranges in parentheses)

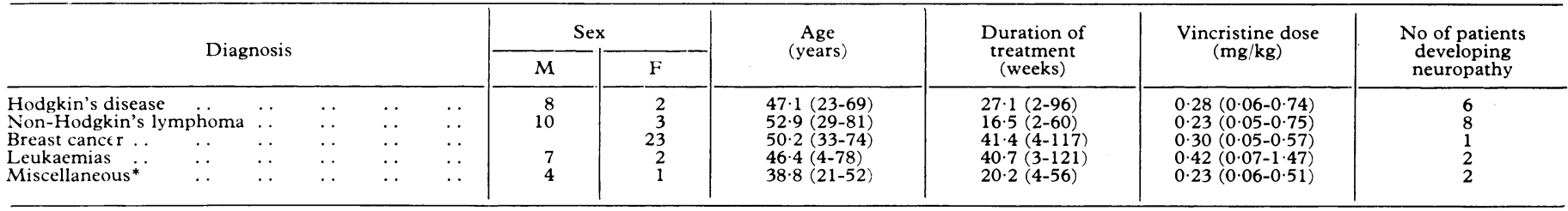

* Malignant melanoma, rectal carcinoma, bronchial carcinoma, undifferentiated sarcoma, and anaplastic carcinoma.

the durations of treatment and mean monthly doses of vincristine in patients with and without lymphoma. In both groups the pattern of dosage with time was essentially similar; the mean dose fell as the treatment time increased, since the most intensive therapy was given in the early weeks, and patients who responded well and survived for

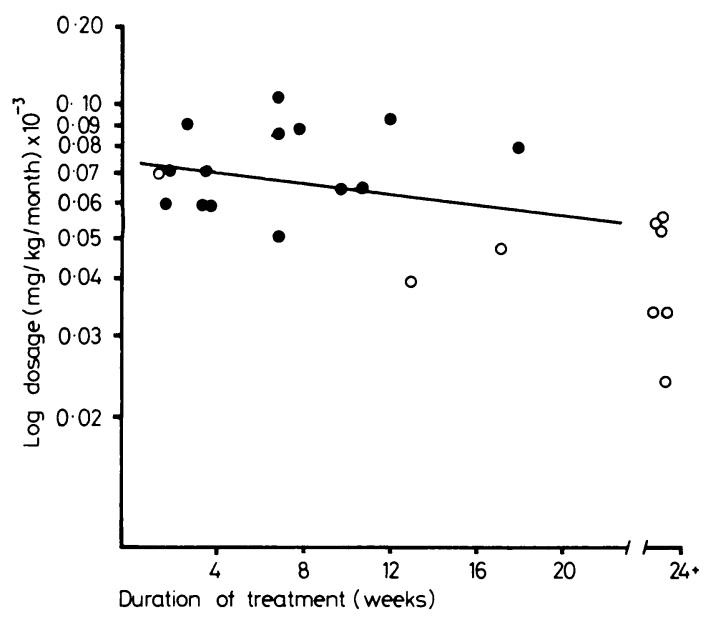

FIG 1-Duration of treatment and mean monthly dose of vincristine in 23 patients with lymphoma. Closed and open circles represent patients with and without vincristineinduced neuropathy respectively. $y=-0.0046 \times+1.8740$.

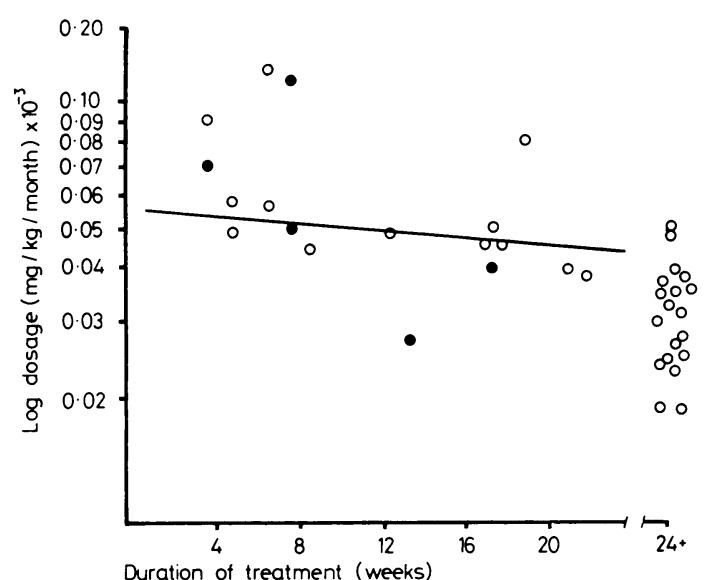

FIG 2-Duration of treatment and mean monthly dose of vincristine in 37 patients with malignant disease other than lymphoma. Closed and open circles represent patients with and without vincristine-induced neuropathy respectively. $y=-0.0042 x+1.7562$

long periods received fewer doses in the later stages. The difference between the doses received by the two groups was not significant after six months: the normal-theory linear model test gave a value of $0.15(P>0.4)$. Neuropathy was far more common in patients with lymphoma than in other patients, despite comparable dosage (figs 1 and 2). In both groups neuropathy tended to occur early in treatment when doses were relatively high. Of the 19 patients who developed neuropathy, 17 did so within the first three months of treatment and seven within the first month.

During the first month of treatment patients with lymphoma were far more likely to develop neuropathy than patients without lymphoma receiving comparable doses of vincristine (fig 3). Doses given in the first month varied, and on average patients who developed neuropathy received slightly higher doses than those who did not (mean $0.082 \mathrm{mg} / \mathrm{kg}$ and $0.073 \mathrm{mg} / \mathrm{kg}$ respectively), but the difference was

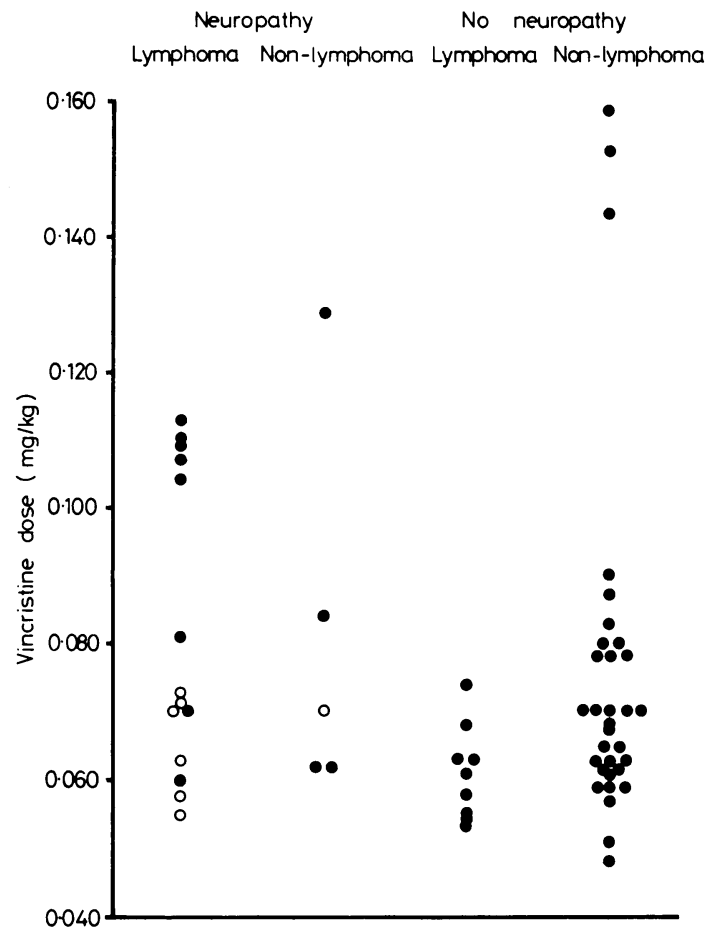

FIG 3-Distribution of patients with lymphoma or other malignant disease according to vincristine dose in first month of treatment. Open circles represent patients who developed neuropathy within first month of treatment.

not significant $(t=1.353 ; \mathrm{DF}=58 ; 0 \cdot 1>\mathbf{P}>0.05)$. The difference between doses received in the first month by patients with lymphoma compared with other patients was not significant either (mean 0.074 $\mathrm{mg} / \mathrm{kg}$ and $0.077 \mathrm{mg} / \mathrm{kg}$ respectively; $t=0.468 ; \mathrm{DF}=58 ; \mathrm{P}>0.3$ ). Vincristine-induced neuropathy in patients with lymphoma seemed to be not only disease-related but also dose-related, for all those who received more than $0.075 \mathrm{mg} / \mathrm{kg}$ in the first month developed neuropathy. This effect was less apparent in the patients without lymphoma; in the first month some received very high doses which were not associated with neuropathy (fig 3).

\section{LIVER INFILTRATION}

Seventeen ( $41 \%$ ) of the 41 patients without neuropathy had clinical and either biochemical or histological evidence or both of liver infiltration by malignant disease. Six $(32 \%)$ of the 19 patients with neuropathy had liver disease. The difference was not significant $\left(\chi^{2}=\right.$ $0.54 ; \mathrm{DF}=58 ; \mathrm{P}>0.4)$. Liver disease was found in $10(43 \%)$ of the 
23 patients with lymphoma and $13(35 \%)$ of the 37 patients with other malignant disease. This difference was not significant $\left(\chi^{2}=0.42\right.$; $\mathrm{DF}=58 ; \mathrm{P}>0.5$.

\section{OTHER DRUGS}

All drugs received by the patients in addition to vincristine, including cytotoxic agents given previously or concurrently, were examined in detail. In patients with neuropathy the following drugs were used with vincristine: cyclophosphamide, mustine, procarbazine, doxorubicin, 5-fluorouracil, prednisolone, metoclopramide, prochlorperazine, diazepam, nitrazepam, and allopurinol. Patients who did not develop neuropathy received all the above drugs except mustine and procarbazine and, in addition, methotrexate, cytarabine, mercaptopurine, asparaginase, and carmustine. Drugs given to patients with lymphoma were studied especially carefully but there was no difference in the medication received by those who developed neuropathy compared with those who did not.

\section{Discussion}

The incidence of vincristine-induced neuropathy was higher in patients with lymphoma $(61 \%)$ than in those with other malignant diseases $\left(14^{\circ}\right)$, despite comparable vincristine dosage in the two groups. Though many patients received comparatively large cumulative doses of vincristine over long periods without side effects, most of those who developed neuropathy did so early in the course of treatment after relatively small total doses of the drug. This may have been because the most intensive treatment was given in the early weeks, and we therefore examined the doses received during this period. Most workers have found vincristine-induced neuropathy to be doserelated, ${ }^{2-7}$ but our patients with neuropathy did not receive significantly higher doses than those without neuropathy during the first month, nor did the patients with lymphoma receive significantly higher doses than the others. Indeed, at comparable doses, far more patients with lymphoma developed neuropathy than did the other patients. Furthermore, although higher doses received in the first month were invariably associated with neuropathy in the patients with lymphoma, the highest doses were given to leukaemic patients, who did not develop neuropathy.

Our findings suggest that susceptibility to the neuropathic effects of vincristine in patients with lymphoma is not only doserelated but also disease-related. Development of neuropathy was clearly related to vincristine since the symptoms appeared within a few weeks of starting treatment and all improved when the drug was withdrawn. Many factors other than dosage may influence the development of neuropathy, such as age differences between the groups; the incidence of carcinomatous neuropathy in the various types of malignancies treated with vincristine; the incidence of liver infiltration; and the effects of other drugs given in combination with vincristine.

Age did not seem to be a factor in our patients, as those who developed neuropathy were slightly but not significantly younger than those who did not. The incidence of carcinomatous neuropathy is low in both breast cancer and Hodgkin's disease (between 1 and $2^{\circ},{ }_{0}$ ). ${ }^{89}$ Thus a predisposition to carcinomatous neuropathy is unlikely to explain the widely different incidences of vincristine neurotoxicity in our two groups of patients. Furthermore, pathological and electromyographical (EMG) findings are different in the two types of neuropathy. ${ }^{2} \times$ In one of our patients with Hodgkin's disease EMG studies showed axonal degeneration, typical of vincristine neuropathy but not of carcinomatous neuropathy, in which segmental demyelination is more characteristic. Patients with impaired liver function are particularly sensitive to the neurotoxic effects of vincristine, probably because of impaired uptake or excretion of the drug, ${ }^{4}$ or both. Nevertheless, the incidence of overt liver disease in the lymphoma group was similar to that in other patients, so this does not explain the increased incidence of vincristine neuropathy in the former group.

Certain cytotoxic drug combinations used in treating the patients with lymphomas-for example, MOPP (mustine, vincristine (Oncovin), procarbazine, and prednisolone); COP (cyclophosphamide, vincristine, and prednisolone); and CHOP (cyclophosphamide, doxorubicin (hydroxydaunorubicin), vincristine, and prednisolone)-were not used in the other patients, and in these particular combinations vincristine could be especially neurotoxic, although a high incidence of neurotoxicity does occur in patients treated with vincristine alone. ${ }^{1+}$ Nevertheless, interaction with other drugs may modify the pharmacokinetics or metabolism of vincristine, thereby affecting the toxicity.

We do not know why patients with lymphoma are apparently so prone to vincristine-induced neurotoxicity. The drug is nevertheless so useful in managing such patients that this predisposition to neurotoxicity should not be regarded as a contraindication to its use. Nevertheless, patients with lymphoma who are receiving vincristine should be observed especially carefully for the onset of neuropathy, and the drug should always be withdrawn if there is evidence of progressive neurotoxicity.

We thank Dr John Williams, of the Lister Hospital, Dr Christophe Wicks, of Eli Lilly and Company, and Dr Peter Watkins, of King's College Hospital, for encouragement and advice; Dr John Durston, of the Whittington Hospital, for carrying out the EMG studies; D Anthony Atkinson, of Imperial College, for statistical advice; Mrs Sally Knight and her staff, of the Lister Hospital library, for help with the references; and $\mathrm{Mr} \mathrm{C}$ Titmus, of the Lister Hospital photographic department, for the figures.

\section{References}

${ }^{1}$ Holland, J F, et al, Cancer Research, 1973, 33, 1258.

2 Sandler, S G, Tobin, W, and Henderson, E S, Neurology (Minneapolis), 1969, 19, 367.

${ }^{3}$ Roeser, H P, et al, British fournal of Haematology, 1975, 30, 233.

4 Shaw, R K, and Bruner, J A, Cancer Chemotherapy Reports, 1964, 42, 45.

${ }^{5}$ Bradley, W G, et al, Fournal of the Neurological Sciences, 1970, 10, 107.

${ }^{6}$ Casey, E B, et al, Brain, 1973, 96, 69.

7 Hardisty, R M, McElwain, T J, and Darby, C W, British Medical fournal, $1969,2,662$.

${ }^{8}$ Henson, R A, Modern Trends in Neurology, No 5, ed D Williams, p 209. London, Butterworths, 1970.

${ }^{9}$ Croft, P B, and Wilkinson, M, Brain, 1965, 88, 427.

(Accepted 30 December 1977) 